\title{
Effect of surface hydrophobicity of therapeutic protein loaded in polyelectrolyte nanoparticles on transepithelial permeability
}

\author{
ANA MIKLAVŽIN ${ }^{1,2}$ \\ MATEJA CEGNAR ${ }^{2}$ \\ JANEZ KERČ $\check{C}^{1,2}$ \\ JULIJANA KRISTL ${ }^{1 *}$ \\ ${ }^{1}$ Faculty of Pharmacy, University of \\ Ljubljana, SI-1000 Ljubljana, Slovenia \\ ${ }^{2}$ Lek Pharmaceuticals d.d., Sandoz \\ Development Center Slovenia, 1526 \\ Ljubljana, Slovenia
}

Accepted May 25, 2018

Published online June 21, 2018

\begin{abstract}
Oral delivery of protein drugs is greatly limited by low hydrophobicity, an important determinant for intestinal epithelial permeation and bioavailability. Herein, surface properties of recombinant erythropoietin were investigated using the fluorescent dye bis-ANS to monitor relative hydrophobicity for correlation with permeabilities with Caco-2 cells. At various $\mathrm{pHs}$, bis-ANS fluorescence intensity indicated different surface hydrophobicities of erythropoietin molecules. Erythropoietin incorporated in chitosan or chitosantrimethylchitosan (CS-TMC) nanoparticles prepared by polyelectrolyte complexation and ionotropic gelation with tripolyphosphate also showed different surface hydrophobicities. Chitosan nanoparticles with erythropoietin provided the most hydrophobic surface, followed by free erythropoietin (in water) and that loaded into CS-TMC nanoparticles. Chitosan nanoparticles were more effective than CS-TMC nanoparticles for permeation of erythropoietin across Caco-2 cell monolayers; the lowest permeability was shown by erythropoietin itself. Thus, hydrophilic protein molecules complexed with polyelectrolytes can provide more hydrophobic surfaces that enhance transepithelial permeability. This bis-ANS method also provides valuable information for the design of polyelectrolyte nanoparticules for oral delivery of protein drugs.
\end{abstract}

Keywords: erythropoietin, bis-ANS fluorescence intensity, chitosan, Caco-2 model, membrane permeability, oral drug delivery

Recent advances in DNA recombinant technology have allowed the production of therapeutic proteins on an industrial scale. These have advantages over conventional small-molecule drugs since they can provide more specific, effective and safer treatments. Hence, therapeutic proteins represent the future of mainstream drugs $(1,2)$.

\footnotetext{
*Correspondence; e-mail: julijana.kristl@ffa.uni-lj.si
} 
The majority of currently marketed peptide and protein drugs are administered via injection although such administration is less acceptable to the patient due to pain and discomfort that can frequently lead to low patient compliance. However, although a highly attractive approach, oral delivery of proteins remains a significant challenge in the design of drug delivery systems because of their unfavourable physicochemical properties. Major limitations for effective oral protein drug bioavailability (which can frequently be $<1 \%$ ) include their relatively high molecular mass, hydrophilic nature, instability due to variations in $\mathrm{pH}$ and enzymes in the gastrointestinal tract (including the natural microbiota), and their poor mucosal and epithelial permeability (3-6). Indeed, as the benefits of therapeutic proteins continue to increase, there will be a growing need for improved oral drug delivery systems.

Nanoparticles (NPs) composed of polymers or lipids have emerged as a major driver among delivery systems for oral administration of protein drugs (7-9). In particular, encapsulation of a protein into such carriers can protect protein drugs from acidic and enzymatic degradation, control their release, and enhance their absorption in the small intestine (7). Integrity of an encapsulated protein has a crucial impact on its pharmacological efficiency. Many and varied approaches to improving encapsulation and drug stability have been investigated (8-10). The parameters of NPs that favour permeation through the barriers in the gut include small particle size, colloidal stability, and suitable hydrophilic/ hydrophobic surface properties (8). It is generally agreed that absorption increases with a decreasing particle diameter. Studies on polystyrene latex particles of $50 \mathrm{~nm}$ to $3 \mu \mathrm{m}$ in diameter have revealed that maximum absorption occurs with particles of 50 to $100 \mathrm{~nm}$, while particles $>1 \mu \mathrm{m}$ are trapped in the lymphoid follicles of the ileum (11).

NPs with a more hydrophobic surface can cross the epithelial barrier more efficiently than those with a hydrophilic surface, although hydrophobic NPs are less stable and show lower mucopermeability (12). To ensure both features, a suitable hydrophilic/hydrophobic balance needs to be provided $(3,8,13,14)$. From the perspective of nanoparticle-membrane interactions, many studies have demonstrated different effects of hydrophobic NPs on the lipid bilayers (14). These studies have defined the key factor for improved permeability as the shift in the hydrophilic to more hydrophobic character of protein drugs. Thus, the hydrophobic character of NPs regulates their entry into epithelial cells and their transition across and exit from these cells on the basolateral side, and hence their entry into the systemic circulation $(13,15,16)$.

Characterization of the surface hydrophobicity of free protein molecules or proteins incorporated in NPs is thus particularly relevant in the design of drug delivery systems. Hydrophobicity of a protein surface depends not only on the chemistry of the underlying amino acids and functional groups of matrix polymers, but also on the precise chemical pattern and topographical context presented by the surface. Characterization of such context-dependent hydrophobicity at nanoscale resolution is not a trivial task (17). In general, the hydrophobicity of composed particles has been determined by indirect methods (e.g., partition coefficients or $\log P$, thin layer chromatography, various computational methods) $(3,13)$. These measurements constitute a limitation for rapid evaluation of protein drug molecules in their native form. For in-depth analysis, fluorescent dyes offer an analytical alternative for determination of hydrophobicity in terms of pharmaceutical formulations. 
Such dyes include 1-anilinonaphtalene-8-sulfonate (ANS), 4,4'-bis-ANS (bis-ANS), Nile red, thioflavin T, dye 9-(dicyanovinyl)-julolidine and Congo red, which can interact noncovalently with protein surfaces. In this context, ANS and its dimeric analogue bis-ANS have been amongst the most frequently used dyes for protein characterization (18). Fluorescence of these dyes is sensitive to their environment, with respect to polarity, viscosity and temperature. Although all of these dyes interact hydrophobically or electrostatically with solutes (e.g., proteins), it has been shown for bis-ANS that hydrophobic interactions dominate over electrostatic interactions $(18,19)$. Thus, in the development of composed NPs, these dyes can provide a way of easily determining surface hydrophobicity. In addition, it is important to investigate the effect of protein or nanoparticle polarity on Caco-2 cell monolayers, since they closely mimic the biological properties of the intestinal epithelial cells (20-22). Permeation of free protein drugs and those loaded into NPs in terms of their surface hydrophobicity has not been studied yet.

Recombinant human erythropoietin (EPO) was selected as the model protein drug. It is a glycoprotein hormone, which is the principal regulator of red blood cell formation (23). Due to the required high doses of parenterally administered EPO, severe side effects have been reported for cancer patients taking EPO and receiving chemotherapy and/or radiation therapy (24). Therefore, there is an unmet need to develop new and improved EPO delivery systems for its oral administration, which might then allow low-dose long-term therapy.

In the present study, surface properties of EPO molecules in solution and those incorporated into NPs were investigated using the fluorescent dye bis-ANS to determine its relative surface hydrophobicity influencing the delivery of EPO across the intestinal epithelial barrier. The hypothesis was that the nanoparticle formation by complexation of charged EPO molecules with oppositely charged polyelectrolyte, i.e., chitosan (CS) and/or trimethylchitosan (TMC), has a decisive consequence on the surface hydrophobicity and on permeability through the Caco-2 cells. The study could provide a protocol that can be used to determine the hydrophobicity and predict the permeability of new protein drugs and to design nanoparticulate delivery systems for their oral administration.

\section{EXPERIMENTAL}

\section{Materials}

Recombinant human erythropoietin (herein EPO) was kindly donated by Sandoz Biopharmaceuticals Mengeš, Slovenia. Low molecular weight chitosan (50-190 kDa; deacetylation degree 75-85\%; viscosity 20-200 mPa s in $1 \%(\mathrm{~m} / \mathrm{V})$ acetic acid solution) and sodium tripolyphosphate (TPP) were from Sigma-Aldrich, USA. N-TMC with $47 \%$ quaternization and not $\mathrm{O}$-methylated was synthesized and characterized using ${ }^{1} \mathrm{H}$ NMR. The extrinsic fluorescence dye bis-ANS was from Molecular Probes, USA. Low molecular mass marker fluorescein was from Sigma Aldrich, USA. The Caco-2 cell line was from the American Tissue Culture Collection, USA. Dulbecco's modified eagle's medium (DMEM), trypsin, heat-inactivated foetal bovine serum, L-glutamine, non-essential amino acids, and bovine 
serum albumin were from Sigma-Aldrich, USA. Antibiotic/antimycotic solutions and phosphate-buffered saline (PBS) were from Invitrogen, USA.

Permeability experiments were performed in 12-well plates (Transwell, Costar 3460; Costar, UK) on polyester permeable supports (membrane growth area $1.12 \mathrm{~cm}^{2}$; pore size $0.4 \mu \mathrm{m}$; Costar, UK). Antibodies and reagents for ELISA were from R\&D Systems (Minneapolis, USA). All other chemicals used were of analytical grade.

\section{Preparation of erythropoietin-loaded nanoparticles}

NPs were prepared under mild conditions using the polyelectrolyte complexation and ionotropic gelation method $(5,10,25)$. For the EPO-loaded NPs, a solution of EPO $(2.67 \mathrm{mg}$ $\left.\mathrm{mL}^{-1}\right)$ was initially prepared in phosphate buffer $\left(50.0 \mathrm{mmol} \mathrm{L}-1 \mathrm{NaHPO}_{4}, 150.0 \mathrm{mmol} \mathrm{L}^{-1}\right.$ $\mathrm{NaCl}$, adjusted with $0.5 \mathrm{~mol} \mathrm{~L}^{-1} \mathrm{NaOH}$ to $\mathrm{pH} 7.4$ ), of which $0.225 \mathrm{~mL}$ was added dropwise to $2 \mathrm{~mL}$ of CS or a mixture of CS and TMC (CS-TMC ratio 1:1) solution (2 $\mathrm{mg} \mathrm{mL}^{-1}$ in 0.25 $\%(V / V)$ aqueous acetic acid, $\mathrm{pH} 4.6)$, at $25^{\circ} \mathrm{C}$ for $30 \mathrm{~min}$, under magnetic stirring (300 rpm). Then $0.900 \mathrm{~mL}$ of TPP solution $\left(1 \mathrm{mg} \mathrm{mL}^{-1}\right.$ in water) was added dropwise into the dispersion and stirred for $45 \mathrm{~min}$ to obtain the final colloidal dispersion of CS/EPO/TPP and CS-TMC/EPO/TPP NPs. These were thus composed of a polymer or a mixture of polymers (CS and/or TMC) $1.28 \mathrm{mg} \mathrm{mL}^{-1}$, EPO $0.192 \mathrm{mg} \mathrm{mL}^{-1}$ and TPP $0.288 \mathrm{mg} \mathrm{mL}^{-1}$. In the same way, empty NPs were prepared, where EPO was replaced by phosphate buffer (pH 7.4). The obtained dispersion of the NPs showed the characteristic Tyndall effect.

\section{Characterization of nanoparticles}

The NPs were characterized according to their hydrodynamic size, polydispersity index and surface zeta potential using Zetasiser Nano ZS; Malvern Instruments, UK. These measurements were carried out at least in triplicate, at $25^{\circ} \mathrm{C}$, using a He-Ne laser $\left(\lambda=633 \mathrm{~nm}\right.$ ) with back-scatter detection (scattering angle, $\left.173^{\circ}\right)$. Data were collected using the Zetasizer software (version 6.20) as the hydrodynamic size (using the Stokes-Einstein equation), polydispersity index, and zeta potential (using the Smoluchowski approximation for the Henry equation). Laser light scattering measurements were used to investigate whether aggregation occurred and to follow dispersion instability.

A scanning electron microscope (SEM, JSM-7001F; Jeol, Japan) was used to evaluate the nanoparticle morphology, with an acceleration voltage of $1.5 \mathrm{kV}$ and a secondary electron detector. Scanning electron microscopy images were taken of the freshly prepared NPs after they were dried in a vacuum dryer (Termo Scientific 36185; Fischer Scientific, USA), at $25^{\circ} \mathrm{C}$ for $24 \mathrm{~h}$, with the samples deposited on double-sided carbon tape (diameter, $12 \mathrm{~mm}$; Oxford Instruments, UK).

\section{Determination of loading capacity of erythropoietin in nanoparticles}

The amount of EPO associated with the NPs represents the loading capacity $(L C)$. This is the difference between the total EPO initially used to prepare the NPs and the amount of non-associated EPO after nanoparticle separation, expressed as a percentage of the total nanoparticle mass, as defined in Eq. (1): 


$$
L C(\%)=\frac{(\text { total amount of EPO-free EPO in supernatant })}{\text { mass of nanoparticles }} \times 100
$$

The free EPO content in the supernatant was determined after ultracentrifugation of $1.5 \mathrm{~mL}$ of nanoparticle dispersion at $128,307 \times \mathrm{g}$ for $20 \mathrm{~min}$, using reverse-phase HPLC (Waters HPLC system; Waters Corporation, USA) with a C18 column (YMC-Pack ODS-AQ; $150 \times 4.6 \mathrm{~mm} ; 3 \mu \mathrm{m})$. Mobile phase A was $10 \%$ acetonitrile in $0.1 \%$ aqueous trifluoroacetic acid, and mobile phase B was $90 \%$ acetonitrile in $0.1 \%$ aqueous trifluoroacetic acid. Gradient elution (flow rate $1 \mathrm{~mL} \mathrm{~min}^{-1} ; 35^{\circ} \mathrm{C}$ ) went from 35 to $65 \%(V / V)$ buffer B in buffer A, providing five column volumes over $30 \mathrm{~min}$ of elution. Detection was at 215 and $280 \mathrm{~nm}$.

\section{Determination of surface hydrophobicity using fluorescent dye}

The bis-ANS dye was used for characterization of the surface hydrophobicity of EPO, either alone (free in solution) or loaded in NPs. Fluorescence emission spectra of bis-ANS in different media were recorded to evaluate the impact of medium polarity and $\mathrm{pH}$ on surface hydrophobicity. The stock solution of EPO $\left(2.67 \mathrm{mg} \mathrm{mL}^{-1}\right)$ was added into water, phosphate buffer $\mathrm{pH} 7.4$ and $0.25 \%(V / V)$ acetic acid, and into media with NPs to achieve the same final concentration of $0.19 \mathrm{mg} \mathrm{mL}^{-1} \mathrm{EPO}$ as in NPs. To evaluate surface characteristics of the EPO-loaded NPs, the fluorescence emission spectra were recorded for EPO in solution and loaded into NPs (CS/EPO/TPP; CS-TMC/EPO/TPP), all with the same medium composition and at the same $\mathrm{pH}$. Into $180 \mu \mathrm{L}$ of these samples, $20 \mu \mathrm{L} 50 \mu \mathrm{mol} \mathrm{L}^{-1}$ bis-ANS was added to achieve the final concentration of bis-ANS $5 \mu \mathrm{mol} \mathrm{L}^{-1}$. Fluorescent intensity of bis-ANS in different samples was measured using a UV-VIS spectrofluorimeter (Safire2, Tecan Austria GmbH, Austria). The excitation wavelength was $385 \mathrm{~nm}$ and emission scans were recorded from 420 to $650 \mathrm{~nm}$, with slits of $1 \mathrm{~nm}$. The Z-position was set to 11,700 $\mu \mathrm{m}$, and a gain of 65 was used. Under these conditions, the inner filter effect was negligible.

\section{Determination of EPO permeability in the Caco-2 cell monolayer}

For transport experiments, Caco-2 cells were seeded and cultured in 12-well permeable support plates at a seeding density of $1 \times 10^{5}$ cells/well in a volume of $0.5 \mathrm{~mL}$ DMEM supplemented with $10 \%(V / V)$ foetal bovine serum, $1 \%(V / V)$ L-glutamine, $1 \%(V / V)$ nonessential amino acids and $1 \%(V / V)$ antibiotics/antimycotics. The cells were grown in an atmosphere containing $5 \% \mathrm{CO}_{2}$ at $37^{\circ} \mathrm{C}$ for 21 days, with the medium changed every 2 to 3 days. One hour before the experiment, the growth medium was changed to permeation medium: Hank's balanced salt solution, buffered with $30 \mathrm{mmol} \mathrm{L}^{-1} \mathrm{~N}$-(2-hydroxyethyl) piperazine- $\mathrm{N}$-(2-ethanosulfonic acid) (HEPES) at $\mathrm{pH}$ 5.6, and the cells were left to equilibrate.

Transepithelial electrical resistance of the Caco-2 cell monolayer and permeability experiments were performed after 3 weeks of Caco-2 cell culturing (cell passages 48-58) on a permeable support in Transwell plates. Tightness of the cell monolayer was tested by measuring TEER (Millicell-ERS volt-ohm meter, Millipore, Billerica, USA), in triplicate. TEER measurements were performed before and after each permeability experiment to check the integrity of the cell monolayer. Tightness was expressed as a percentage of the 
initial TEER. For permeability experiments, complete culture medium of the Caco-2 cell was removed from both apical and basolateral compartments and the cells were rinsed with DMEM without supplements (transport medium). To $1.5 \mathrm{~mL}$ of individual test samples, $0.399 \mathrm{~mL}$ of transport medium was added to achieve the final EPO concentration of $0.15 \mathrm{mg} \mathrm{mL}^{-1}$. In the apical compartment, $0.5 \mathrm{~mL}$ of such tested samples and $1.5 \mathrm{~mL}$ transport medium were added into the basolateral compartment. Permeation with the tested samples was performed under the same conditions as when the Caco-2 cells were grown. Then, $110 \mu \mathrm{L}$ samples were taken from the basolateral compartment at 30, 60, 120 and 180 min, which were always replaced by fresh transport medium. As the negative control for membrane tightness, the paracellular permeability marker fluorescein was used as previously described (24). When monolayer integrity during the permeability experiment was diminished, the test sample was removed, cells were washed and returned to complete cell culture medium, left to regenerate for $24 \mathrm{~h}$, and then TEER was measured again. All the permeability experiments were performed as three repeats.

\section{Apparent permeability coefficient and enhancement ratio calculation}

The apparent permeability coefficient $\left(P_{\text {app }}\right)$ of EPO across the Caco-2 cell monolayer was determined and calculated from the measured concentration of EPO in the apical and basolateral compartments at determined times according to the following equation derived from Fick's law for steady state and sink conditions:

$$
\operatorname{Papp}(\mathrm{cm} / \mathrm{s})=\frac{d Q}{d t} \times \frac{1}{A \times c_{0}}
$$

where the ratio $d Q / d t\left(\mathrm{~mol} \mathrm{~s}^{-1}\right)$ was calculated from the linear part of the curve (i.e., increasing mass in the basolateral compartment over time), $A\left(\mathrm{~cm}^{2}\right)$ is the permeation area of the membrane, and $c_{0}\left(\mathrm{~mol} \mathrm{~L}^{-1}\right)$ is the initial concentration of EPO in the apical compartment. EPO concentration was measured on both sides, apical and basolateral, to exclude its possible metabolic degradation in Caco-2 cells.

As a more immediate measure of these effects of chitosan and CS-TMC NPs on EPO permeability in this Caco-2 cell model, enhancement ratios were also calculated. These defined $P_{\text {app }}$ of EPO in nanoparticle formulations $\left(P_{\text {app }} \mathrm{EPO}_{\text {formulation }}\right)$ relative to $P_{\text {app }}$ of the free $\mathrm{EPO}\left(P_{\text {app }} \mathrm{EPO}_{\text {free }}\right)$, as defined in Eq. (3):

$$
\text { Enhancement ratio }=\left(P_{\text {app }} \mathrm{EPO}_{\text {formulation }} / P_{\text {app }} \mathrm{EPO}_{\text {free }}\right)
$$

\section{Quantification of erythropoietin}

EPO concentrations of collected samples were measured using an in-house sandwich ELISA protocol, with commercially available antibodies and reagents. Microtiter plates pre-coated with an anti-EPO antibody were incubated with $100 \mu \mathrm{L}$ of samples at $25^{\circ} \mathrm{C}$ for $1 \mathrm{~h}$, on a horizontal orbital microplate shaker at $500 \mathrm{rpm}$. After careful removal of all medium, the wells were washed with $400 \mu \mathrm{L}$ wash buffer and incubated with $100 \mu \mathrm{L}$ anti- 
human EPO detection antibody, for $1 \mathrm{~h}$, under the same conditions as the testing formulations. The wells were then washed three times with $400 \mu \mathrm{L}$ wash buffer, and incubated with $200 \mu \mathrm{L}$ horseradish peroxidase conjugated anti-rabbit IgG antibody for $1 \mathrm{~h}$. This was followed by another wash. The substrate solution containing $0.35 \mathrm{mg} \mathrm{mL}^{-1} 3,3^{\prime}, 5,5^{\prime}$-tetramethylbenzidine was added, with incubation for 20 to $25 \mathrm{~min}$. The reaction was stopped by the addition of $0.1 \mathrm{~mL} 2 \mathrm{M}$ sulphuric acid solution, and the absorbances at 450 and 600 $\mathrm{nm}$ (for correction) were measured (Synergy HT plate reader, BioTek Instruments, USA). EPO concentrations were calculated from the prepared standard curve, which showed linearity in a concentration range of 0.015 to $1.0 \mathrm{ng} \mathrm{mL}^{-1}\left(R^{2}=0.9999\right)$.

Fluorescein was analysed using a microplate reader (FLUOstar Galaxy, BMG Labtechnologies $\mathrm{GmbH}$, Germany) at 485 and $530 \mathrm{~nm}$ as excitation and emission wavelengths, respectively. Fluorescein concentration was calculated from the prepared standard curve, which showed linearity in a concentration range of $0.1 \mathrm{ng} \mathrm{mL}^{-1}$ to $0.1 \mu \mathrm{g} \mathrm{mL} \mathrm{L}^{-1}$ with the correlation coefficient 0.9998 .

\section{Data analysis}

Representative data of two or three independent experiments are presented as means \pm standard deviation, with four to six replicates for each experiment. The SPSS 10.0 for Windows (SPSS, Chicago, IL) statistical software package was used for data analysis. Statistical significance for all the tests was accepted at a level of $p<0.05$.

\section{RESULTS AND DISCUSSION}

The motivation for this study was to design and test a highly sensitive method for determination of surface hydrophobicity of protein drugs alone and loaded into NPs. Surface hydrophobicity is an important property of NPs, which influences the fate, transport and bioavailability of protein drugs. Previous studies aimed to investigate protein structural changes, protein adsorption, and conformational changes of protein drugs after their loading into NPs (25). Herein, we have determined and characterized the surface hydrophobicity of the protein drug EPO alone or incorporated into NPs as an approach to improving protein drug permeation through the intestinal epithelial barrier.

\section{Characteristics of nanoparticles}

Polyelectrolyte complexation and ionotropic gelation are the mildest methods of nanoparticle preparation, avoiding the use of harsh processing conditions such as organic solvents, high energy processing procedures and/or surfactants, which are potentially harmful to sensitive biomolecules. In the polyelectrolyte complexation used in the present study, the main ionic interactions were between the positive amino groups of the CS or the mixture of CS-TMC and negative carboxylic residues of EPO amino-acid to form fragile NPs, and later with the phosphate groups of TPP, when stable NPs were made. While this crosslinking takes place, the EPO is also loaded into the matrix of the NPs by electrostatic and hydrophobic interactions (Fig. 1). Nanoparticle formation is primarily entropy driven 

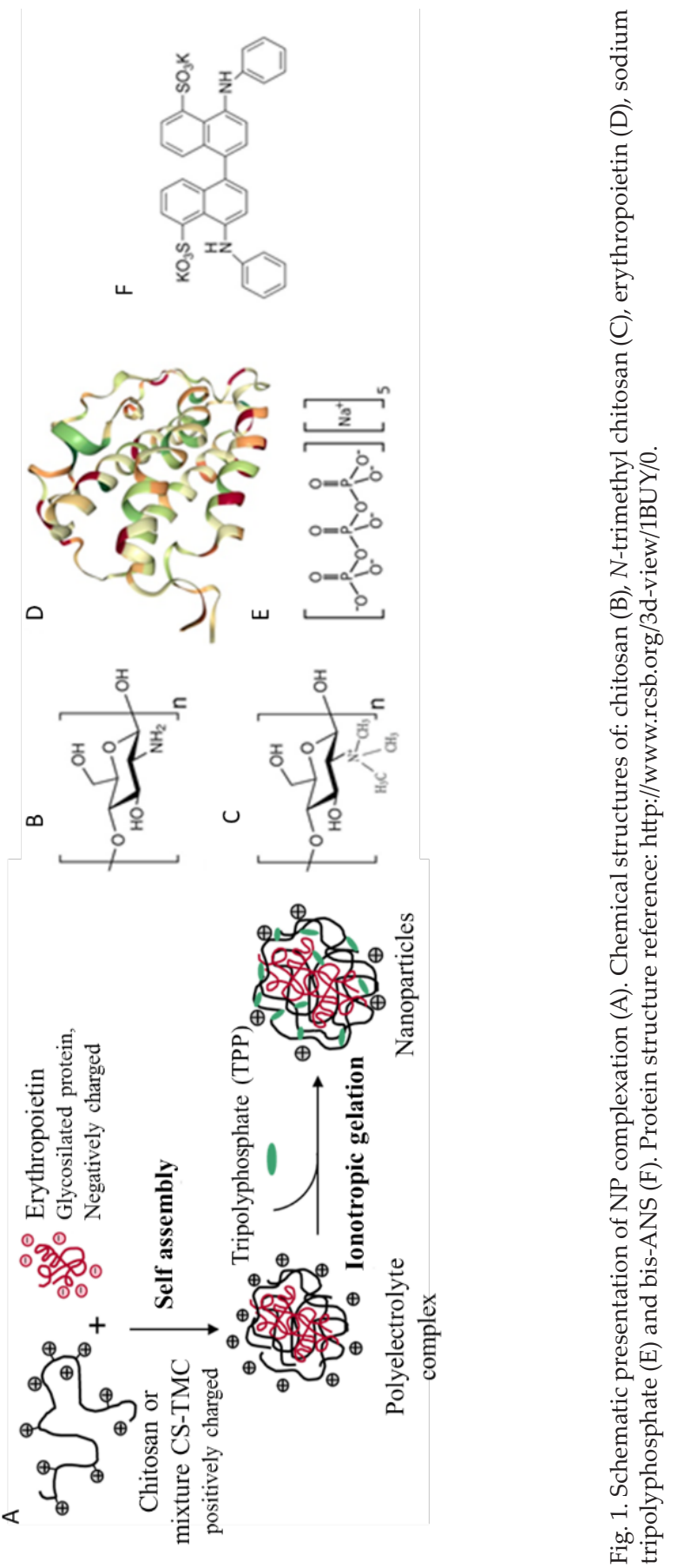
due to the intrinsic physicochemical properties of the polycationic polymers used, and the anionic EPO, and tripolyphosphate, which occur spontaneously upon this dropwise combination under gentle stirring (10). The mean particle size, polydispersity index and zeta potential of the EPO-loaded CS NPs were $300 \pm 35 \mathrm{~nm}, 0.30 \pm 0.022$ and $+30 \pm 3 \mathrm{mV}$, respectively, and for the EPO-loaded CS-TMC NPs were $250 \pm 20 \mathrm{~nm}, 0.31 \pm 0.025$ and $+35 \pm 4 \mathrm{mV}$. Colloidal stabilities defined by the surface zeta potentials were close for both of these

a)

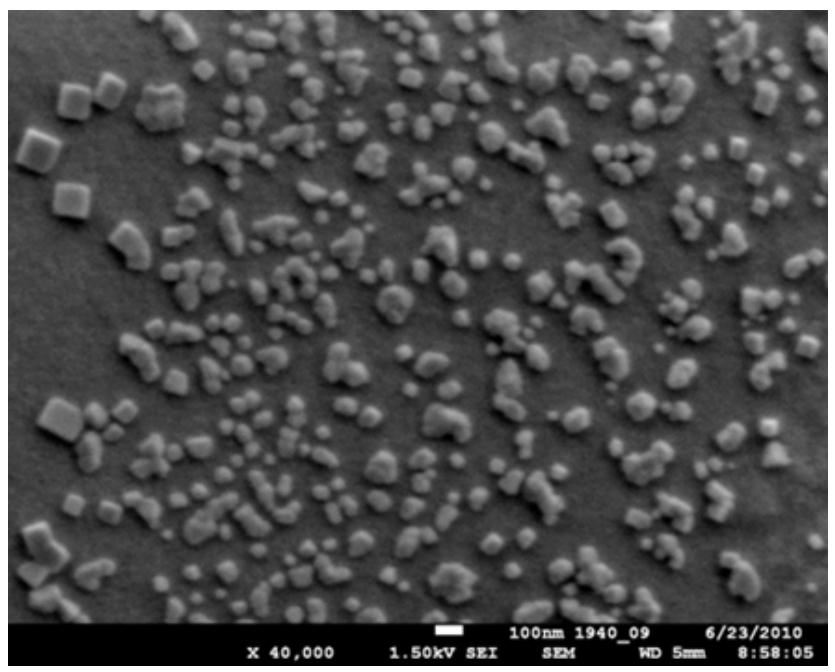

b)

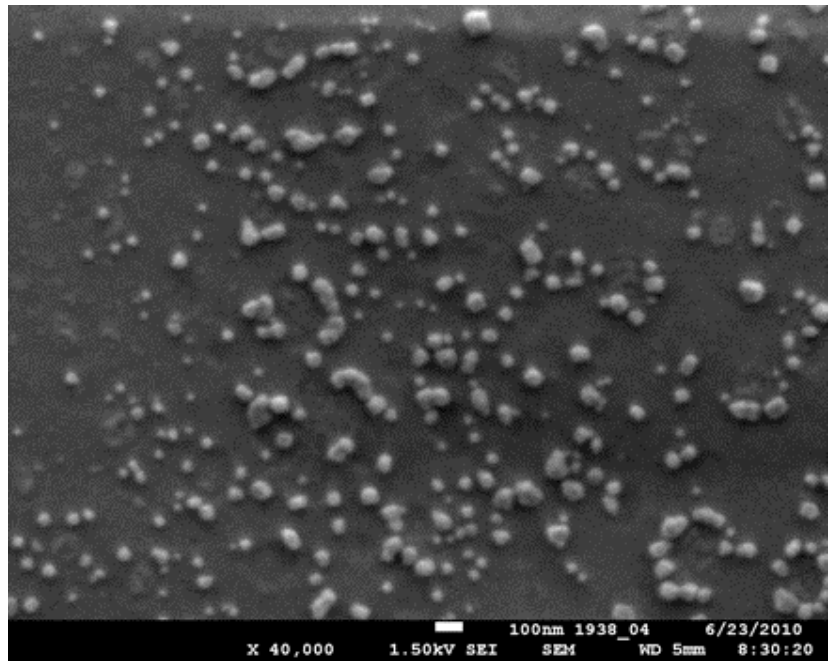

Fig. 2. Representative SEM images of: a) the EPO-loaded chitosan NPs, and b) CS-TMC NPs. Bar size $100 \mathrm{~nm}$. 


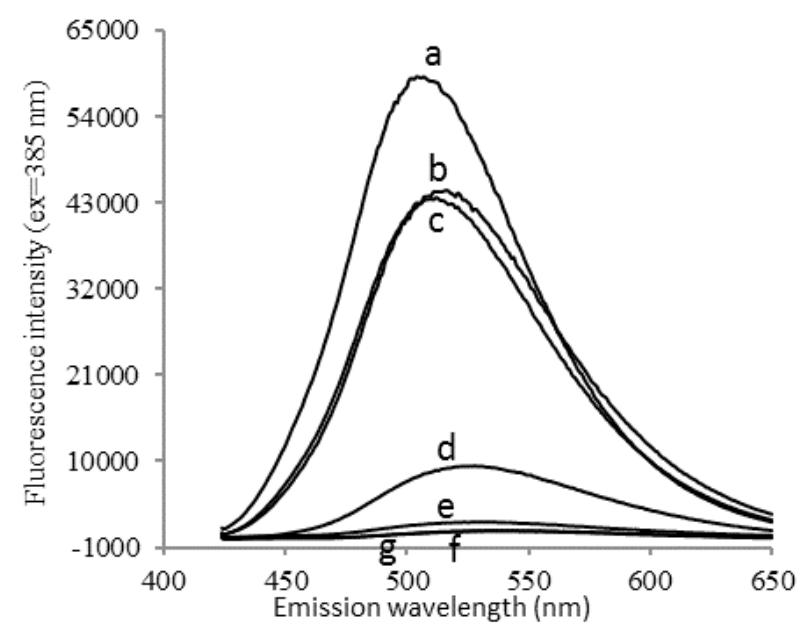

Fig. 3. Bis-ANS fluorescence intensity in different solvents with various polarities: a) acetone, b) methanol, c) absolute ethanol, d) $50 \%(V / V)$ aqueous ethanol, e) phosphate buffer (pH 7.4), f) $0.25 \%(V / V)$ acetic acid, and g) water.

EPO-loaded NPs (range, +27 to $+39 \mathrm{mV}$ ), and therefore these nanoparticle dispersions were stable. The loading efficiency of EPO into CS/EPO/TPP and CS-TMC/EPO/TPP NPs was 62 and $33 \%$, respectively. Lower loading efficiency for CS-TMC/EPO/TPP NPs might be due to the steric obstacles of the methyl groups of TMC.

SEM images of dried NPs showed an irregular and spherical to polyhedral shape and were seen as clusters of individual NPs, mainly due to the drying under vacuum (Fig. 2). However, boundaries between the individual particles were still visible. Estimated sizes of the particles obtained from the SEM images were by about 100 to $150 \mathrm{~nm}$ smaller than those measured using dynamic light scattering. The reason is probably related to the hydration of particles in water for the measurement of the hydrodynamic radius.

\section{Fluorescence intensity of bis-ANS in different media}

Fluorescence intensity of the bis-ANS dye is strongly dependent on the polarity of the media in which it is dissolved, as shown in Fig. 3. The highest fluorescence intensity was observed in acetone, with the lowest dielectric constant of $20.7 \mathrm{As} \mathrm{V}^{-1} \mathrm{~m}^{-1}$, followed by methanol (32.1 As $\left.\mathrm{V}^{-1} \mathrm{~m}^{-1}\right)$ and absolute ethanol $\left(24.5 \mathrm{As} \mathrm{V}^{-1} \mathrm{~m}^{-1}\right)$, which showed comparable intensities despite the different dielectric constants. In $50 \%$ ethanol, bis-ANS showed an even lower intensity compared to absolute ethanol, as expected. In water with a dielectric constant of $80.1 \mathrm{As} \mathrm{V}^{-1} \mathrm{~m}^{-1}$, bis-ANS showed minimal fluorescence intensity (Fig. 3e-g). The data indicate that bis-ANS shows minimal fluorescence in an aqueous environment, but becomes highly fluorescent in apolar, organic solvents with low dielectric constants. These data are in agreement with Hawe et al. (18) and Maurel reports (27). 
A. Miklavžin et al.: Effect of surface hydrophobicity of therapeutic protein loaded in polyelectrolyte nanoparticles on transepithelial permeability, Acta Pharm. 68 (2018) 275-293.

Table I. Zeta potentials of EPO protein molecules in water media with different $p H s$. NP medium contains the same solvents and buffers with the same $\mathrm{pH}$ as used in the prepared NPS

\begin{tabular}{lcc}
\hline EPO medium & $\mathrm{pH}$ & Zeta potential $(\mathrm{mV})$ \\
\hline Phosphate buffer & 7.4 & $-10.6 \pm 0.2$ \\
Water & 7.2 & $-10.4 \pm 0.3$ \\
NPs medium & 5.4 & $-6.7 \pm 0.2$ \\
$0.25 \%(V / V)$ acetic acid & 4.1 & $-3.6 \pm 0.4$ \\
\hline
\end{tabular}

Overall, this evaluation confirms that the bis-ANS fluorescence intensity is inversely correlated with the polarity of the medium, and that it is suitable for the intended investigation.

Hydrophobicity of the erythropoietin protein molecule in media with different $p H s$

The impact of medium $\mathrm{pH}$ on the hydrophobicity of the EPO protein molecule was evaluated in solvents with different $\mathrm{pHs}$, in terms of zeta potentials (Table I). As with EPO, many proteins show a pronounced tendency to ionize amino acid residues at a specific $\mathrm{pH}$, which results in different polarities of the protein surface (27). To evaluate the surface hydrophobicity of EPO alone and loaded in the NPs at different pHs, bis-ANS was used because of its different fluorescence intensities upon contact with various hydrophobic surfaces. EPO in solvents with different $\mathrm{pHs}$ had various negative zeta potentials, and therefore showed different tendencies for interactions with bis-ANS. Fluorescence inten-

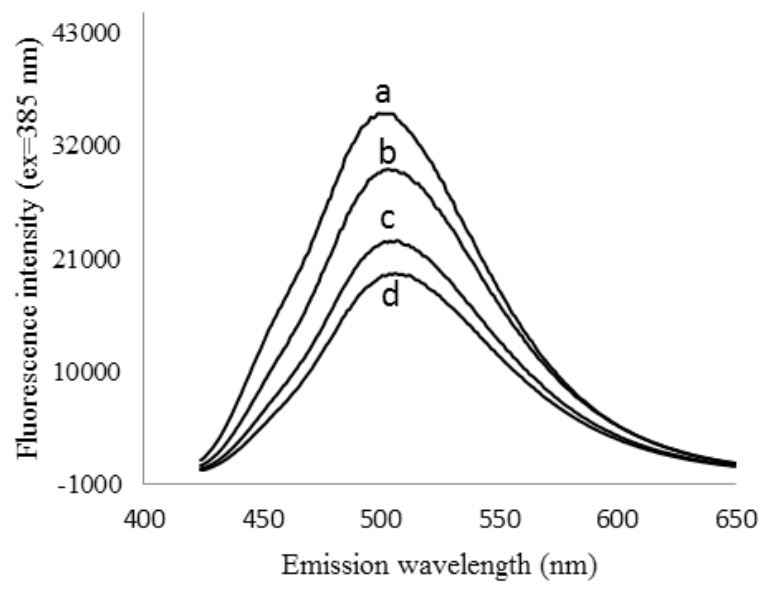

Fig. 4. Bis-ANS fluorescence intensity in the presence of EPO in the media with different pHs: a) 0.25 $\%(V / V)$ acetic acid, b) NPs medium, c) water, and d) phosphate buffer ( $\mathrm{pH} 7.4$ ). 
sity reflects the surface hydrophobicity of EPO, which can be partly affected by the medium $\mathrm{pH}$ due to the exposure of its hydrophobic parts (domains with less charges) on the surface. However, detailed information about bis-ANS binding with EPO can be obtained using nuclear magnetic resonance and circular dichroism. This trend is consistent with the data of Chi et al. (27), who reported that the solution $\mathrm{pH}$ can define the type (positive or negative) and total charge on a protein, thereby affecting the electrostatic interactions and protein hydrophobicity. The number of charged groups on a protein varies according to the $\mathrm{pH}$ of the solution.

Fluorescence intensity of bis-ANS alone in the selected water media with different pHs was negligible, while the intensity changed significantly $(p<0.05)$ after EPO addition, as shown in Fig. 4. Bis-ANS showed its highest fluorescence intensity in the presence of EPO in an acidic environment of $0.25 \%(V / V)$ acetic acid, followed by the NP medium, and then even lower at almost neutral $\mathrm{pH}$ (i.e., phosphate buffer, water). Explanation of these data can be found in the different ionization steps of the EPO molecule at different $\mathrm{pHs}$. As the isoelectric point of EPO is 4.4 to 4.8 (23), the effect of ionized groups is the smallest close to this pHs, and therefore the hydrophobicity of EPO is the highest (Table I, Fig. 4a). The further the $\mathrm{pH}$ is from the isoelectric point, the more ionized groups will be found on the surface of the globular protein (i.e., greater zeta potential), and the surface will be less hydrophobic and more hydrophilic, which is reflected in lower fluorescence intensity (Fig. $4 b-d)$.

\section{Hydrophobicity of the erythropoietin loaded nanoparticles}

The different fluorescence intensities of bis-ANS in the presence of free EPO and EPOloaded into NPs and the respective controls are shown in Figs. 5a and 5b. Here, the EPOloaded into CS/EPO/TPP NPs showed the highest fluorescence intensity compared to free EPO under the same conditions and solvent composition. Fluorescence intensities of the empty CS/TPP NPs and chitosan polymer in NPs medium were negligible compared to the samples with EPO (Fig. 5a).

Fig. $5 \mathrm{~b}$ shows fluorescence intensity of the EPO loaded into CS-TMC/EPO/TPP NPs. Here, the highest fluorescence intensity was seen for free EPO in the medium for NPs, which was thus higher than for the EPO loaded into CS-TMC/EPO/TPP NPs (Fig. 5b).

Comparison of fluorescence intensities between EPO loaded into both types of NPs indicated higher hydrophobicity for CS/EPO/TPP than for CS-TMC/EPO/TPP NPs. This was most likely due to the charges on NP surfaces as a consequence of the different EPO complexation to each of these polymers. Hydrophobicity probably reflected fewer interactions between TMC and EPO compared to CS.

The CS and CS-TMC mixture, recorded solely in NP medium, and also after crosslinking with TPP to form empty NPs, resulted in low values of bis-ANS fluorescence, indicating a highly charged NP surface. Our experimental data fit the Cooper et al. (25) report, where they concluded that interactions of proteins with polyelectrolyte carriers reduce the dielectric environment of the protein, which can result in increased fluorescence intensity, and thus reflects higher surface hydrophobicity after its complexation into NPs. However, the fluorescence intensity of these CS-TMC/EPO/TPP NPs was slightly higher than the empty CS-TMC/TPP NPs (i.e., 11,406 compared to 7,607). The lower hydrophobicity of CS- 
TMC/EPO/TPP NPs might be a consequence of the remaining charges at CS-TMC polymers due to the low EPO loading ability.

In addition, this study opened more options to increase the protein drug loading in polyelectrolyte nanoparticles if the type and structure of the polymer, surface charge density and $\mathrm{pH}$ value of the medium are selected properly. Consequently, local structural features, such as the arrangement of polymer segments in their "bound state" on a $0.5-10$ $\mathrm{nm}$ scale, need to fit the binding domain of the protein drug. Thus, higher loading ability promotes electrostatic interactions and shifts the protein molecule polarity.

Based on fluorescence intensities, it can be concluded that the EPO loaded into CS/ $\mathrm{EPO} / \mathrm{TPP}$ NPs shows the most hydrophobic character, followed by free EPO in medium and then the EPO loaded into CS-TMC/EPO/TPP NPs. In further experiments, the impact of hydrophobicity on the permeability of the model drug EPO loaded into both types of NPs was evaluated in vitro.

\section{Monitoring Caco-2 cell monolayer integrity by transepithelial electrical resistance}

One of the mechanisms of NPs to facilitate the permeation of protein drugs is to compromise an epithelial monolayer. Therefore, the monolayer tightness was examined using the transport of the paracellular permeability marker, fluorescein, at the same concentration as EPO $\left(0.15 \mathrm{mg} \mathrm{mL}^{-1}\right)$ to confirm the suitability of this Caco-2 model for testing permeability. The $P_{\text {app }}$ for fluorescein was consistent throughout the transport experiments, with a mean of $3.1 \pm 1.5 \times 10^{-8} \mathrm{~cm} \mathrm{~s}^{-1}$ and was lower than those from other laboratories (22). In addition, the monitoring of the Caco- 2 cell monolayer was evaluated by measuring TEER, and the return to the initial TEER after permeation. In general, TEER is used to define the tightness of the junctions between cells, and thus the disturbance of the junctions is also reflected in changes to TEER. TEER was monitored at the start of each experiment, during the 3-h experimental incubations, and then $24 \mathrm{~h}$ after them. In all cases where the TEER was $>2 \mathrm{k} \Omega \mathrm{cm}^{2}$ at the start, this was accepted as the appropriate monolayer integrity for permeability experiments. In general, TEER values ranging from 0.15 to $1 \mathrm{k} \Omega$ $\mathrm{cm}^{2}$ have been reported $(7,21-23)$. Higher TEER measurements along with lower $P_{\text {app }}$ of fluorescein indicate that the Caco-2 cell monolayers used in our experiments are tighter than those reported elsewhere (22).

Following the 3-h incubations of Caco-2 monolayers with apical application of EPO loaded into CS/EPO/TPP or CS-TMC/EPO/TPP NPs, significant TEER decreases were observed. While the free EPO in NP medium did not affect the TEER, after its complexation into CS/EPO/TPP NPs it reduced it by $80 \%$, and into CS-TMC/EPO/TPP NPs by $77 \%$ (Table II). The recovery period for TEER after the removal of free EPO in NP medium and EPO loaded into CS and CS-TMC NPs (i.e., 24 h) saw TEER restoration to $70 \%$ of the initial value. These data did not match those of Sadeghi et al. (28), who reported that the decrease in TEER for CS NPs was only about $15 \%$ after $5 \mathrm{~h}$ of incubation with Caco-2 cells. This might be due to the lower zeta potential of NPs $(+15.9 \pm 0.5 \mathrm{mV})$ and smaller particle size (around $200 \mathrm{~nm}$ ) prepared by Sadeghi et al. (28). It is known that the surface charge density of NPs and their size also affect their ability to interact with the components of tight junctions and compromise them to allow particle absorption. 
a)

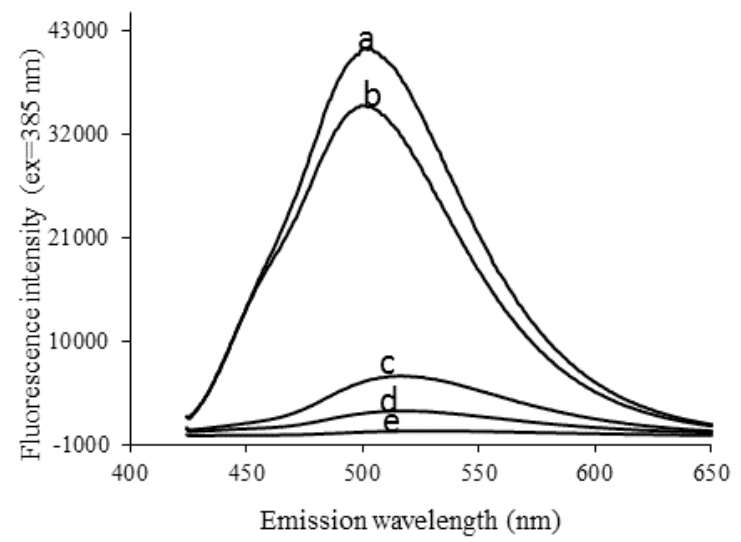

b)

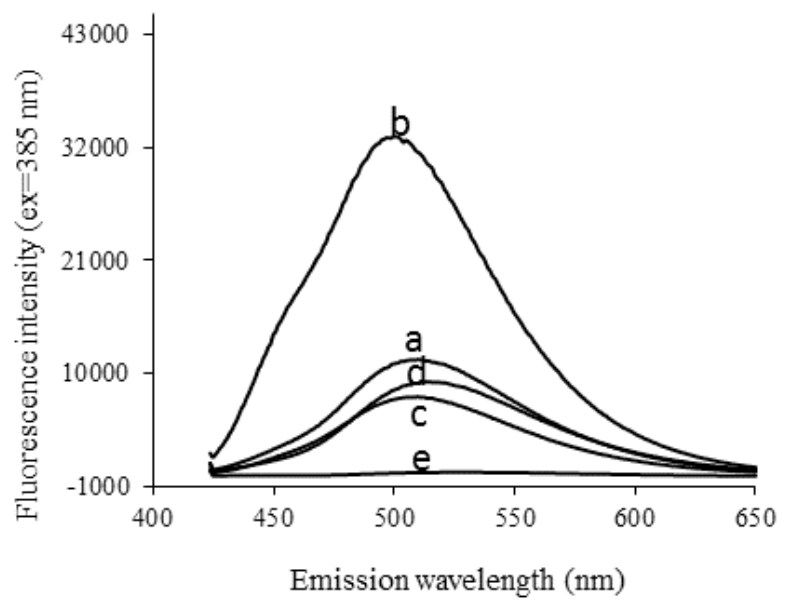

Fig. 5. Bis-ANS fluorescence intensity for EPO free and loaded into the CS and CS-TMC nanoparticle systems: a) CS/EPO/TPP NPs (a), free EPO in NPs medium (b), empty CS/TPP NPs (c), chitosan polymer in NP medium (d), and NP medium alone (e); b) CS-TMC/EPO/TPP NPs (a), free EPO in NP medium (b), empty CS-TMC/TPP NPs (c), CS-TMC polymer mixture in NP medium (d), and NP medium alone (e). All samples were recorded in the same dispersion media (NP medium), having a $\mathrm{pH}$ of 5.4.

Permeability of erythropoietin protein molecules and nanoparticles through Caco-2 cells

In previous sections, hydrophobicity of the investigated samples and the membrane integrity were presented, while here the impact of the hydrophobic nature of these EPO samples on their ability to cross the Caco-2 cell monolayers was investigated. The data from these permeation experiments are given as the calculated apparent permeability coefficient, $P_{\text {app }}$ and enhancement ratio, as shown in Table II. 
A. Miklavžin et al.: Effect of surface hydrophobicity of therapeutic protein loaded in polyelectrolyte nanoparticles on transepithelial permeability, Acta Pharm. 68 (2018) 275-293.

Table II. EPO formulations and their effects on TEER of the Caco-2 cell model after 3-h treatments, and after 24-h recovery, and the apparent permeability coefficient

\begin{tabular}{|c|c|c|c|c|c|}
\hline \multirow{2}{*}{ Sample } & \multirow{2}{*}{$\begin{array}{l}\text { Concentrations } \\
\left(\mathrm{mg} \mathrm{mL}^{-1}\right)\end{array}$} & \multicolumn{2}{|c|}{$\begin{array}{l}\text { Transepithelial electrical } \\
\text { resistance (\% initial) }\end{array}$} & \multirow{2}{*}{$\begin{array}{c}P_{\text {app }} \text { EPO } \\
\left(\mathrm{cm} \mathrm{s}^{-1}\right)\end{array}$} & \multirow{2}{*}{$\begin{array}{c}\text { Enhancement } \\
\text { ratio }\left(P_{\text {app }}\right. \\
\text { sample/ } P_{\text {app }} \\
\text { EPO })\end{array}$} \\
\hline & & $\begin{array}{c}\text { Following } 3 \mathrm{~h} \\
\text { treatment }\end{array}$ & $\begin{array}{l}\text { Following } 24 \mathrm{~h} \\
\text { recovery }\end{array}$ & & \\
\hline EPO in NPs medium & 0.15 & 100 & 100 & $4.09 \pm 0.2 \mathrm{E}-10$ & I \\
\hline CS/EPO/TPP & $1.0 / 0.15 / 0.225$ & 20 & 70 & $2.60 \pm 0.3 \mathrm{E}-07$ & 636 \\
\hline CS-TMC/EPO/TPP & $1.0 / 0.15 / 0.225$ & 23 & 70 & $1.71 \pm 0.1 \mathrm{E}-08$ & 42 \\
\hline
\end{tabular}

$P_{\text {app }}-$ calculated apparent permeability coefficient

The mean $P_{\text {app }}$ defined higher permeability across the Caco-2 cell monolayers for EPO loaded into CS NPs, followed by EPO loaded into CS-TMC NPs, and then free EPO. Enhancement ratios as their $P_{\text {app }}$ relative to that of free EPO in NPs medium (Table II) were 636 for CS/EPO/TPP NPs but only 42 for CS-TMC/EPO/TPP NPs. Thus, these CS NPs were much more effective in promoting the permeation of EPO across the Caco-2 cell monolayer than the CS-TMC NPs. While for the EPO-loaded CS NPs this parallels their higher hydrophobicity, the EPO-loaded CS-TMC NPs had lower hydrophobicity compared to that of free EPO in NP medium. This indicates the reverse, since despite this lower hydrophobicity, the permeability of EPO-loaded into CS-TMC/EPO/TPP was higher than for free EPO in NP medium. To conclude, encapsulations of protein drugs into polyelectrolyte NPs increased protein hydrophobicity and reduced their size, thus allowing them to pass the epithelial barrier.

Although Li et al. (13) and Mohammed et al. (29) reported that hydrophobic NPs can undergo inclusion into the membrane, whereas semihydrophilic NPs will adsorb onto the membrane, this is not the only mechanism to improve protein absorption. NP parameters that can affect cellular uptake, protein binding and translocation of the NPs through the cell are also size, chemical composition, shape, surface charge density and their aggregation (15). However, at this level, definition of the detailed mechanisms behind the permeabilities of these NPs with these mixtures of polymers will require further, more detailed analyses. Furthermore, although most studies have reported CS enhancement of intestinal paracellular permeation, some studies have also shown that it can directly affect both paracellular and transcellular permeation. Thus, they defined CS and TMC (either in solution or as NPs) as permeation enhancers to improve the permeability through the intestinal epithelium of macromolecule drugs $(6,28,29)$. They also indicated that the highly positively charged TMC provides increased permeability of macromolecular drugs across the intestinal cells, compared to CS (29). Data from the present study show that the permeability of CS-TMC/EPO/TPP NPs was lower than for CS/EPO/TPP NPs although the EPO-loaded into CS-TMC NPs had higher positive net charge, which might better interact with the negatively charged interior of tight junctions and thus open the paracellular route. 
Furthermore, to investigate the influence of NPs prepared in this study on the permeability through the mucus and intestinal barriers, additional methods (e.g., confocal microscopy) and in vitro cell model (e.g., Caco-2/MT 29 co-cultured cells) should be used. There Caco-2 cells simulate the intestinal epithelium and its tightness, the MT29 cells secret mucus and simulate an intestinal mucus layer with lower TEER. So far, we have proved the impact of hydrophobic effects of these polyelectrolyte NPs on the permeability of EPO protein drugs through the intestinal barrier, but the issues of mucus permeation remain open. The difference in surface charge density of investigated NPs would probably also affect the permeation through the mucus, as also reported by de Sousa (30).

\section{CONCLUSIONS}

The present study is focused on investigating the surface hydrophobicity of free EPO, as a model protein drug, and loaded into NPs, to determine any correlations with permeation through an intestine epithelium model. Surface hydrophobicity was determined using the extrinsic fluorescent dye bis-ANS, which showed specific polarity-sensitive binding. These data show that under a variety of polarities and $\mathrm{pHs}$ of the medium, EPO undergoes surface changes that reflect different surface hydrophobicities. The highest surface hydrophobicity of EPO was observed at around $\mathrm{pH}$, where it had the lowest ionization. A direct correlation between fluorescence intensity of bis-ANS and polarity confirmed an experimental metric for prediction of relative surface hydrophobicity. CS/EPO/ TPP NPs showed higher hydrophobicity than CS-TMC/EPO/TPP NPs, and when evaluated in vitro with Caco-2 cell monolyers, also higher $P_{\mathrm{app}}$, while free EPO in NPs medium showed the lowest $P_{\text {app }}$. The study confirms that surface polarity of nanosized particles represents an important parameter in protein permeability in the intestine. We can thus conclude that these data suggest that the complexation of hydrophilic protein drug molecules with polyelectrolytes may form more hydrophobic NPs enhancing protein drug permeability through the epithelial layer. Finally, this concept can be used with practically any type of protein drugs where polyelectrolyte complexation principles are taken into account. Combination of bis-ANS and the permeability study also provide a valuable approach that will help in the development phase of new therapeutic protein drugs and their delivery systems, since this approach requires relatively little sample consumption and less time in comparison with other biophysical methods.

Acknowledgments. - The authors thank the Department of Pharmaceutical Chemistry, Faculty of Pharmacy, University of Ljubljana, for chitosan chemical modification and the Department of Molecular Biology and Nanotechnology, National Institute of Chemistry, Slovenia, to carry out experiments on Caco-2 cells. The study was supported by the Slovenian Research Agency through the research programme P1-0189. 


\section{REFERENCES}

1. J. K. Ryu, H. S. Kim and D. H. Nam, Biotechnol. Bioprocess Engin., 17 (2012) 900-911; http:// doi.org/10.1007/s12257-012-0095-1

2. J. Wang, V. Yadav, A. L. Smart, S. Tajiri and A. W. Basit, Toward oral delivery of biopharmaceuticals: an assessment of the gastrointestinal stability of 17 peptide drugs, Mol. Pharm. 12 (2015) 966-973; http://doi.org/10.1021/mp500809f

3. O. Zupančič and A. Bernkop-Schnürch, Lipophilic peptide character - What oral barriers fear the most?, J. Control. Release 255 (2017) 242-257; http://doi.org/10.1016/j.jconrel.2017.04.038

4. K. Park, I. C. Kwan and K. Park, Oral protein delivery: current status and future prospect, React. Funct. Polym. 71 (2011) 280-287; http://doi.org/10.1016/j.reactfunctpolym.2010.10.002

5. D. Vllasaliu, R. Exposito-Harris, A. Heras, L. Casettari, M. Garnett, L. Illum and S. Stolnik, Tight junction modulation by chitosan nanoparticles: Comparison with chitosan solution, Int. J. Pharm. 400 (2010) 183-193; http://doi.org/10.1016/j.ijpharm.2010.08.020

6. G. Camenisch, J. Alsenz, H. V. Waterbeemd and G. Folkers, Estimation of permeability by passive diffusion through Caco-cell monolayers using the drugs' lipophilicity and molecular weight, Eur. J. Pharm. Sci. 6 (1998) 317-324; http://doi.org/10.1016/S09280987(97)10019-7

7. B. F. Choonara, Y. E. Choonara, P. Kumar, D. Bijukumar, L. C. du Toit and V. Pillay, A review of advanced oral drug delivery technologies facilitating the protection and absorption of protein and peptide molecules, Biotechnol. Adv. 32 (2014) 1269-1282; http://doi. org/10.1016/j.biotechadv.2014.07.006

8. T. Jung, W. Kamm, A. Breitenbach, E. Kaiserling, J. X. Xiao and T. Kissel, Biodegradable nanoparticles for oral delivery of peptides: is there a role for polymers to affect mucosal uptake? Eur. J. Pharm. Biopharm. 50 (2000) 147-160; http://doi.org/10.1016/S09396411(00)00084-9

9. P. Ahlin Grabnar and J. Kristl, The manufacturing techniques of drug-loaded polymeric nanoparticles from preformed polymers, J. Microencaps. 28 (2011) 323-335; http://doi.org/ 10.3109/02652048.2011.569763

10. J. Mirtič, J. Ilaš and J. Kristl, Influence of different classes of crosslinkers on alginate polyelectrolyte nanoparticle formation, thermodynamics and characteristics, Carbohydrate polymers 181 (2018) 93-102; http://doi.org/10.1016/j.carbpol.2017.10.040

11. A. T. Florence, Nanoparticle uptake by the oral route: Fulfilling its potential? Drug Discovery Today: Technologies 2 (2005) 75-81; http://doi.org/10.1016/j.ddtec.2005.05.019

12. L. Yin, J. Ding, C. He, L. Cui, C. Tang and C. Yin, Drug permeability and mucoadhesion properties of thiolated trimethyl chitosan nanoparticles in oral insulin delivery, Biomaterials 30 (2009) 5691-5700; http://doi.org/10.1016/j.biomaterials.2009.06.055

13. Y. Li, X. Chen and N. Gu, Computational investigation of interaction between nanoparticles and membranes: Hydrophobic/hydrophilic effect, J. Phys. Chem. B. 112 (2008) 1664716653; http://doi.org/10.1021/jp8051906

14. J. Renukuntla, A. D. Vadlapudi, A. Patel, S. H. S. Boddu and A. K. Mitra, Approaches for enhancing oral bioavailability of peptides and proteins, Int. J. Pharm. 447 (2013) 75-93; http://doi.org/10.1016/j.ijpharm.2013.02.030 
15. C. Contini, M. Schneemilch, S. Gaisford and N. Quirkedoi, Nanoparticle-membrane interactions, J. Exper. Nanosci. 13 (2018) 62-81; http://doi.org/10.1080/17458080.2017.1413253

16. Y. Xiao, M. R. Wiesner, Characterization of surface hydrophobicity of engineered nanoparticles, J. Hazard. Mater, 215-216 (2012) 146-151; http://doi.org/10.1016/j. jhazmat.2012.02.043

17. J. A. Patel and S. Garde, Efficient method to characterize the context-dependent hydrophobicity of proteins, Phys. Chem. B. 118 (2014) 1564-1573; http://doi.org/10.1021/jp4081977

18. A. Hawe, M. Sutter and W. Jiskoot, Extrinsic fluorescent dyes as tools for protein characterization, Pharm. Res. 25 (2008) 1487-1499; http://doi.org/10.1007/s11095-007-9516-9

19. D. Matulis, R. Lovrien, 1-Anilino-8-naphthalene sulfonate anion-protein binding depends primarily on ion pair formation, Biophys. J. 74 (1998) 422-429; http://doi.org/10.1016/ S0006-3495(98)77799-9

20. B. Press and D. Di Grandi, Permeability for intestinal absorption: Caco-2 assay and related issues, Curr. Drug Metab. 9 (2008) 893-900; http://doi.org/10.2174/138920008786485119

21. I. Hubatsch, E. G. Ragnarsson and P. Artursson, Determination of drug permeability and prediction of drug absorption in Caco-2 monolayers, Nat. Protoc. 2 (2009) 2111-2119; http:// doi.org/10.1038/nprot.2007.303

22. K. Berginc, S. Žakelj, L. Levstik, D. Uršič and A. Kristl, Fluorescin transport properties across artificial lipid membranes, Caco-2 cell monolayers and rat jejunum, Eur. J. Pharm. Biopharm. 66 (2007) 281-285; http://doi.org/10.2016/j.ejpb.2006.10.023

23. M. Cegnar, B. Podobnik, S. Caserman, M. Homar and J. Kerc, EPO Compositions for Oral Administration, WO2015/032973 (A1), 12 Mar 2015.

24. M. Marušič, T. Zupančič, G. Hribar, R. Komel, G. Anderluh and S. Caserman, The Caco-2 cell culture model enables sensitive detection of enhanced protein permeability in the presence of N-decyl-ß-D-maltopyranoside, New Biotechnologies 30 (2013) 507-515; http:// doi.org/10.1016/j.nbt.2013.05.008

25. C. L. Cooper, P. L. Dubin, A. B. Kayitmazer and S. Turksen, Polyelectrolyte-protein complexes, Curr. Opin. Colloid. Interface Sci. 10 (2005) 52-78, http://doi.org/10.1016/j.cocis.2005.05.007

26. P. Maurel, Relevance of dielectric constant and solvent hydrophobicity to the organic solvent effect in enzymology, J. Biol. Chem. 253 (1978) 1671-1683.

27. E. Y. Chi, S. Krishnan, T. W. Randolph and J. F. Carpenter, Physical stability of proteins in aqueous solution: mechanism and driving forces in non-native protein aggregation, Pharm. Res. 20 (2003) 1325-1336; http://doi.org/10.1023/A:1025771421906

28. A. M. M. Sadeghi, F. A. Dorkoosh, M. R. Avadi, M. Weinhold, A. Bayat, F. Delie, R. Gurny, B. Larijani, M. Rafiee-Tehrani and H. E. Junginger, Permeation enhancer effect of chitosan and chitosan derivatives: Comparison of formulations as soluble polymers and nanoparticulate systems on insulin absorption in Caco-2 cells, Eur. J. Pharm. Biopharm. 70 (2008) 270-278; http://doi.org/10.1016/j.ejpb.2008.03.004.29

29. M. A. Mohammed, J. T. M. Syeda, K. M. Wasan and E. K. Wasan, An overview of chitosan nanoparticles and its application in non-parenteral drug delivery, Pharmaceutics 9 (2017) E53; http://doi.org/10.3390 / pharmaceutics9040053 
30. I. Pereira de Sousa, C. Steiner, M. Schmutzler, M. D. Wilcox, G. J. Veldhuis, J. P. Pearson, C. W. Huck, W. Salvenmoser and A. Bernkop-Schnürch, Mucus permeating carriers: formulation and characterization of highly densely charged nanoparticles, Eur. J. Pharm. Biopharm. 97 (2015) 273-279; http://doi.org/10.1016/j.ejpb.2014.12.024 